\title{
UNCERTAINTY QUANTIFICATION FOR THE TRAILING-EDGE NOISE OF A CONTROLLED- DIFFUSION AIRFOIL
}

\author{
Christophe, J. ${ }^{1}$, Moreau, S. ${ }^{2}$, and Schram, C. ${ }^{1}$ \\ ${ }^{1}$ Dept. of Environmental and Applied Fluid Dynamics, von Karman Institute for Fluid Dynamics, 72 chaussée de Waterloo, \\ Rhodes-St-Genèse, Belgium, 1640, julien.christophe@vki.ac.be \\ ${ }^{2}$ Dépt de Génie Mécanique, Université de Sherbrooke, 2500 Boul. de 1'Université, Sherbrooke QC, Canada, J1K2R1
}

\section{INTRODUCTION}

In modern rotating machines, due to the significant effort put on reducing annoying discrete tones, the broadband noise is an important contribution to the overall noise level, as in fans, turboengines or wind turbines. A key source of broadband noise is the trailing-edge noise, caused by the scattering of boundary-layer pressure fluctuations into acoustic waves at the trailing-edge of any lifting surface. Numerical methods to evaluate this noise are more often using steady RANS computations for computational cost reasons, requiring modelling and introducing then uncertainties. The present study aims at assessing uncertainties associated with the prediction of trailing-edge noise, through an uncertainty quantification (UQ) framework, using RANS computations or conventional LES computations, in order to determine their respective robustness and accuracy.

\section{METHODOLOGY FOR UQ}

The approach to uncertainty quantification (UQ) of airfoil trailing-edge noise is illustrated in Figure 1. The considered case is a Controlled-Diffusion airfoil of chord $C$ placed in the large anechoic wind tunnel in Ecole Centrale de Lyon (LWT), and held by two horizontal side plates. The angle of attack (aoa) is $8^{\circ}$ and the airfoil upstream velocity $U_{0}$ is $16 \mathrm{~m} / \mathrm{s}$, which corresponds to a Reynolds number based on the airfoil chord $R e_{C}=1.6 \times 10^{5}$.

As in previous studies (Moreau et al. 2006), a RANS computation of the complete experimental setup of the large anechoic wind tunnel in Ecole Centrale de Lyon (LWT), including the nozzle and part of the anechoic chamber has been done that captures the strong jet-airfoil interaction and its impact on airfoil loading. The boundary conditions are extracted ( $U$ and $V$ profiles) for a smaller domain embedded in the jet potential core. This final result is obtained by two different procedures, both producing a wall frequency pressure spectrum $\Phi_{p p}$ used in Amiet's theory (Amiet 1976) to predict the far-field sound spectrum $S_{p p}$.

In the first approach, an unsteady LES on the restricted domain with the above extracted velocity profiles, directly yields the trailing edge wall pressure spectrum. The second approach, less expensive but requiring more modeling, uses steady RANS computations on a two-dimensional slice of the restricted domain, with the same boundary condition profiles as for the LES. From this RANS computation, the primitive variables ( $U_{1}, U_{2}, k$ and $\omega$ or $\varepsilon$ depending on the RANS turbulence model) are extracted through a boundary layer profile at the trailing-edge of the airfoil. Those

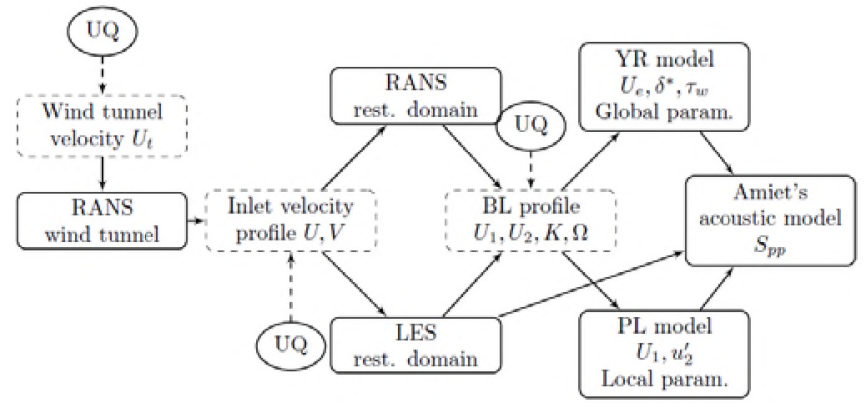

Figure 1. Uncertainty quantification methodology.

variables are then used in the two wall-pressure models investigated in the present study. On the one hand. Rozenberg et al. 2010 proposed a model (YR) only based on global boundary layer parameters from the boundary layer profile, mainly the external velocity $U_{e}$, a boundary layer thickness $\delta$ and the wall shear stress $\tau_{\mathrm{w}}$. On the other hand, the model of Panton \& Linebarger 1974 uses local parameters i.e. the streamwise velocity profile $U_{1}$ and the crosswise velocity fluctuation profile $u_{2}^{\prime}$.

RANS computations are obtained with the solver Ansys Fluent 12, using the Shear-Stress-Transport (SST) $k$ $\omega$ turbulence model. The computational grid is a twodimensional cut perpendicular to the airfoil span of the structured mesh used by Wang et al. 2004. The LES are performed by the CDP code of Stanford University using the dynamic subgrid-scale model. The mesh CDP-B is taken from Moreau et al. 2006 and has 1.5 million cells. The same inflow/outflow conditions are used in the RANS and LES computations and periodic boundary conditions are applied in the spanwise direction for the 3D LES domain.

The uncertainty is introduced at the inlet boundary of the restricted computational domain. The physical variations in the experimental flow measurements are taken into account by selecting a $2.5 \%$ error bound on the streamwise velocity $U$ and a $10 \%$ error bound on the crosswise velocity $V$ around the deterministic numerical solution. A set of 9 velocity inlet profiles are determined using a Clenshaw-Curtis quadrature and the corresponding RANS and LES computations are run. Both components $U$ and $V$ are assumed random variables with uniform distribution within their interval of variation. The stochastic collocation method is used to estimate the uncertainties.

\section{RESULTS}

The flow around the airfoil is illustrated in Figure 2 by iso-surfaces of $Q$ for the LES. As in previous studies 


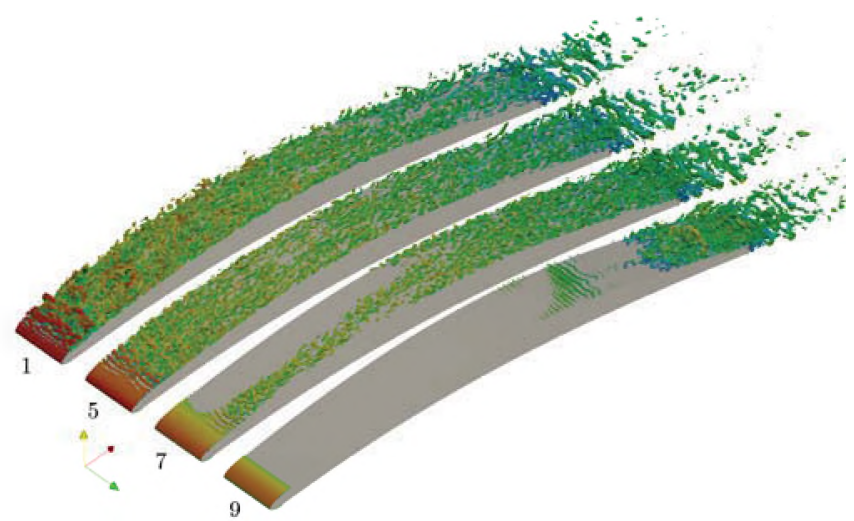

Figure 2. Isosurfaces of the $Q$ factor $\left(Q C^{2} / C_{0}{ }^{2}=2000\right)$ coloured by velocity magnitude for inflow conditions corresponding to LES computations \# 1,5,7,9.

(Wang et al. 2004, Moreau et al. 2006), in LES \#1 and \#5 (reference), small instabilities form close to the reattachment point of the laminar recirculation bubble. The flow tends to re-laminarise toward the mid-chord due to the favorable pressure gradient. When this gradient becomes adverse, the turbulent boundary layer thickens again and larger turbulent structures appear near the trailing-edge. In LES \#7, the acceleration around the leading-edge still yields a weak flow separation at the leading-edge, which is not strong enough to trigger transition over the whole span. Turbulence only develops over a narrow band and only the adverse pressure gradient after mid-chord triggers the full transition and turbulence development. In LES \#9, the acceleration around the leading-edge is no longer strong enough to trigger a flow separation at the leading-edge and no transition occurs before mid-chord. Flow separation occurs beyond mid-chord that triggers the transition close to the trailing edge. This observation is a significant departure from RANS computations where the second recirculation zone beyond mid-chord never occurs for low aoa. This difference is related to the used RANS modelisation that is considering fully turbulent flows and therefore cannot correctly take into account the laminar and transition regions whereas LES calculations do.

From the RANS computations, boundary-layer profiles are extracted near the trailing-edge and the two reconstruction methods are applied to obtain the trailingedge wall-pressure spectra. From the LES computations, the trailing-edge spectrum is directly computed from the wallpressure fluctuations. The wall-pressure spectra are then used in Amiet's theory to compute the radiated far-field sound. The corresponding results together with experimental measurements are shown in Figure 3, in terms of mean and $100 \%$ uncertainty bars. A good agreement with experiments is found for both methods using RANS information. Larger uncertainty bars are found at high frequencies using the YR model due to the large uncertainties involved in wall shearstress determination, on which the model is based at high frequencies. The PL model, not based on the wall shearstress variable, shows less variation at low frequencies but

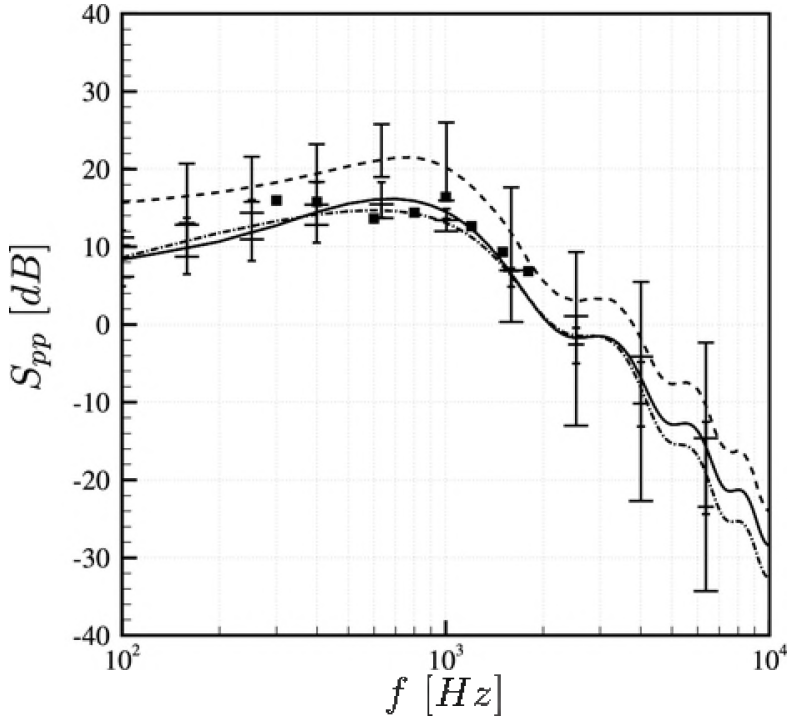

Figure 3. Means and uncertainty bars of far-field acoustic spectra in the mid-span plane above the airfoil $(\theta=90$ ) at $R=$ $2 \mathrm{~m}$ from the trailing edge. (dash-dot and large uncertainty bars) YR model, (plain and small uncertainty bars) PL model, (dash and medium uncertainty bar) LES. (Square) Experiments.

larger uncertainties at low frequencies caused by the low statistical convergence of the Monte-Carlo integration technique used to integrate the boundary-layer profiles. Finally, due to a different flow behaviour in LES \#7 to 9, the LES results are shifted to higher levels by about $8 \mathrm{~dB}$. Consequently, the LES uncertainty bars are also found larger than those of the RANS computations.

\section{REFERENCES}

Amiet, R.K. (1976), "Noise due to Turbulent Flow past a Trailing Edge", J. of Sound and Vib., 47(3), 387-393.

Moreau, S., Neal, D., Khalighi, Y., Wang, M. and Iaccarino, G. (2006) "Validation of Unstructured-Mesh LES of the TrailingEdge Flow and Noise of a Controlled-Diffusion Airfoil." In Proceedings of the Summer Program 2006. Centre for Turbulence Research, Stanford Univ./NASA Ames.

Rozenberg, Y., Moreau, S., Henner, M. and Morris, S. C. (2010) "Fan Trailing-Edge Noise Prediction Using RANS Simulations." In 16th AIAA/CEAS Aeroacoustics Conference. AIAA-2010-3720. Panton, R. L. and Linebarger, J. H. (1974) "Wall Pressure Spectra Calculations for Equilibrium Boundary Layers." J. Fluid Mech., 65(2), 261-287.

Wang, M., Moreau, S., Iaccarino, G. and Roger, M. (2004) "LES Prediction of Pressure Fluctuations on a Low Speed Airfoil." In Annual Research Briefs. Centre for Turbulence Research, Stanford Univ./NASA Ames.

\section{ACKNOWLEDGEMENTS}

The authors would like to thank Stanford University for providing the necessary computational resources. The participation of the first author to the conference is supported through the FP7ECOQUEST project (Grant Agreement no 233541). 